\title{
There is much more than just diseases that underlies frailty in older persons
}

\author{
Matteo Cesari ${ }^{1,2}$ (1)
}

Received: 18 December 2018 / Accepted: 10 May 2019 / Published online: 21 May 2019

(c) Springer Nature Switzerland AG 2019

In this issue of Aging Clinical \& Experimental Research, Gips and colleagues' study aims to optimize the risk prediction of short-term Emergency Department (ED) re-admission in older patients [1]. In a previous work, these authors presented the nomogram they designed for that purpose [2]. The current article examines if the predictive capacity of this nomogram could be improved by adding some variables and constructs. The results outlined here show a minimum additional contribution of comorbidity indexes in the risk prediction of ED re-admission. At the same time, a 10-item frailty score seemed to be irrelevant.

As these investigators explained, there are many possible explanations to these apparently counterintuitive findings. Most importantly, the results seem quite puzzling, especially in view of the fact that the comorbidity indexes (marginally) succeeded where the 10-item frailty score failed. The fact that the Charlson and the Elixhauser's indexes were capable of slightly improving the predictive capacity of the nomogram potentially confutes the hypotheses that the substantially negative results are linked to: (1) a particularly high level of frailty in the population (i.e., determining a sort of ceiling effect), and/or (2) the presence of a pre-existing social support at home. In fact, in both cases, the high number of nosological conditions captured by the comorbidity indexes should have paralleled the individual's increased vulnerability, consequently justifying the need for support at home even before the ED admission.

Personally, I think that the negative findings reported by Gips and colleagues were due to some theoretical and methodological aspects underlying the construct of frailty adopted. First, although the simultaneous presence of

Matteo Cesari

macesari@gmail.com

1 Department of Clinical Sciences and Community Health, University of Milan, Milan, Italy

2 Geriatric Unit, Fondazione IRCCS Ca' Granda Ospedale Maggiore Policlinico, Via Pace 9, 20122 Milan, MI, Italy multiple diseases (or multimorbidity) is not synonymous for frailty, the two conditions are still overlapping. In other words, presumably a person with a high Charlson's index is also likely to be frail (and consequently exposed to an increased risk of negative health-related outcomes [3]). Furthermore, if we consider the theoretical definition of frailty (i.e., a condition characterized by reduced homeostatic reserves determining an increased vulnerability exposing the individual to a high risk of events [4]), it is hard to explain why multimorbidity should not be part of it. After all, diseases are indeed major contributors to the definition of the organism's homeostatic capacity $[5,6]$. Thus, stating that multimorbidity, but not frailty, increases the accuracy of the nomogram, may seem like a terminology/statistical artifice that does not adequately capture the unicity of the organism (in which frailty and multimorbidity share common biological foundations) [7].

Second, frailty was defined here using a 10-item instrument composed by variables that were not included in the original nomogram, but identified as consistently present across several Frailty Indexes available in the literature. By doing so, the authors may have overlooked some methodological points required to design a Frailty Index [8]. In particular, there is not a critical quantity of information that is necessary for a robust computation (i.e., only ten items instead of the minimum requirement of 20). It also seems that the authors thought the Frailty Index was based on a set of pre-defined variables, when one of the strengths of this model is the possibility of generating it by taking advantage of every variable defining a health deficit (i.e., signs, symptoms, diseases, and disabilities). That is, it is not critically important what is introduced in the definition of the Frailty Index, but the mass of information alimenting it. This explains why the authors may have experienced some difficulty in finding similar variables across Frailty Indexes. Indeed, these tools can be different because they refer to a quantitative and not qualitative approach in selecting the constituting variables [9]. It is possible that if the 
Authors had been more flexible in selecting the items constituting their Frailty Index, they would have generated a more comprehensive and stronger tool. This explains why I am concerned that the so-called Frailty Index represents a weak reproduction of the model proposed by Rockwood and Mitnitski [10] and am afraid it explains its null contribution to risk prediction.

The relatively modest capacity of the original nomogram (with or without extra components) to predict the 28-day ED re-admission is another unexplained point. In fact, I think that an area under the curve below 0.7 might be insufficient to support the use of the nomogram for the purposes studied. Why is the nomogram proposed unable to adequately capture the risk of ED re-admission? To my knowledge, the nomogram proposed here does not include a specific section describing the environment which the person lives in or measuring his/her social network. I understand that in the original development of the tool, social factors were not considered in the final model because they were not statistically significant at the preparatory analysis [2]. Information on these factors may nevertheless be crucial to interpret these findings. The presence of a social support system is certainly pivotal in keeping a frail individual at home and preventing ED admissions [11]. The beneficial effects of the most technically perfect intervention will be lost miserably if the frail individual is discharged without an exhaustive evaluation and a coherent social care support system [12].

In conclusion, Gips et al.'s study underlines, once again, the importance of adopting a multidimensional approach when evaluating older persons, that goes beyond traditional paradigms. A large quantity of pertinent information is needed to estimate the risk of an elderly person's re-admission to ED and to design a personalized plan of intervention respectful of his/her needs and resources. Social aspects cannot be ignored, as without them, an individual would be an amorphous mass of biological reactions and arguable nosological labelling. Goethe once wrote: "Tell me whom you consort with and I will tell you who you are". As far as an elderly who is being discharged from ED is concerned, I would say: "Tell me who makes up your social network, and I will tell you if you will be back soon".

\section{Compliance with ethical standards}

Conflict of interest The author declares that he has no conflict of interest.
Research involving human participants and/or animals This article does not contain any studies with human participants or animals performed by the author.

Informed consent This article does not contain any studies with human participants performed by the author.

\section{References}

1. Gips E, Spilsbury K, Boecker C et al (2018) Do frailty and comorbidity indices improves risk prediction of 28-day ED reattendance? Reanalysis of an ED discharge nomogram for older people. Aging Clin Exp Res. https://doi.org/10.1007/s40520-018-1089-4

2. Arendts G, Etherton-Beer C, Jones R et al (2015) Use of a risk nomogram to predict emergency department reattendance in older people after discharge: a validation study. Intern Emerg Med 10:481-487

3. Bahrmann A, Benner L, Christ M et al (2018) The Charlson Comorbidity and Barthel Index predict length of hospital stay, mortality, cardiovascular mortality and rehospitalization in unselected older patients admitted to the emergency department. Aging Clin Exp Res. https://doi.org/10.1007/s40520-018-1067-x

4. Morley JE, Vellas B, Abellan van Kan G et al (2013) Frailty consensus: a call to action. J Am Med Dir Assoc 14:392-397

5. Boyd CM, Ritchie CS, Tipton EF, Studenski SA, Wieland D (2008) From bedside to bench: summary from the american geriatrics society/national institute on aging research conference on comorbidity and multiple morbidity in older adults. Aging Clin Exp Res 20:181-188

6. Guido D, Perna S, Peroni G, et al. (2015) A comorbidity prognostic effect on post-hospitalization outcome in a geriatric rehabilitation setting: the pivotal role of functionality, assessed by mediation model, and association with the Brass index. Aging Clin Exp Res 27:849-856

7. Cesari M, Pérez-Zepeda MU, Marzetti E (2017) Frailty and multimorbidity: different ways of thinking about geriatrics. J Am Med Dir Assoc 18:361-364

8. Searle S, Mitnitski A, Gahbauer E, et al. (2008) A standard procedure for creating a frailty index. BMC Geriatr 8:24

9. Cesari M, Gambassi G, Abellan van Kan G, et al. (2014) The frailty phenotype and the frailty index: different instruments for different purposes. Age Ageing 43:10-12

10. Mitnitski AB, Mogilner AJ, Rockwood K (2001) Accumulation of deficits as a proxy measure of aging. Sci World J 1:323-336

11. Poli S, Cella A, Puntoni M et al (2017) Frailty is associated with socioeconomic and lifestyle factors in community-dwelling older subjects. Aging Clin Exp Res 29:721-728

12. Marzetti E, Sanna T, Calvani R, et al. (2016) Brand new medicine for an older society. J Am Med Dir Assoc 17:558-559

Publisher's Note Springer Nature remains neutral with regard to jurisdictional claims in published maps and institutional affiliations. 